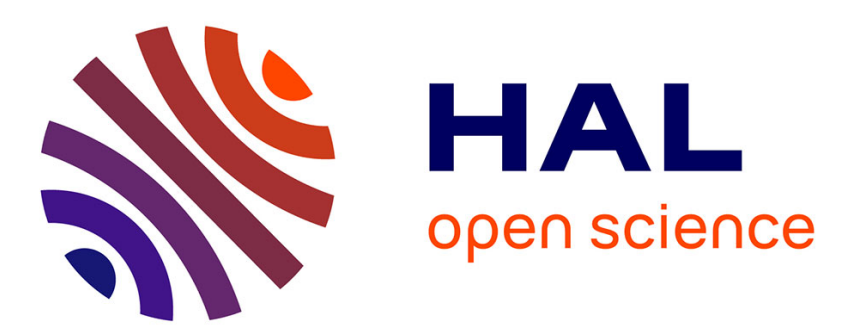

\title{
MIRACULOUS HELICITY RULES, BASES, AND AMPLITUDES FOR VECTOR BOSONS
}

\author{
R. Brown
}

\section{To cite this version:}

R. Brown. MIRACULOUS HELICITY RULES, BASES, AND AMPLITUDES FOR VECTOR BOSONS. Journal de Physique Colloques, 1985, 46 (C2), pp.C2-3-C2-11. 10.1051/jphyscol:1985201 . jpa-00224510

\section{HAL Id: jpa-00224510 https://hal.science/jpa-00224510}

Submitted on 1 Jan 1985

HAL is a multi-disciplinary open access archive for the deposit and dissemination of scientific research documents, whether they are published or not. The documents may come from teaching and research institutions in France or abroad, or from public or private research centers.
L'archive ouverte pluridisciplinaire HAL, est destinée au dépôt et à la diffusion de documents scientifiques de niveau recherche, publiés ou non, émanant des établissements d'enseignement et de recherche français ou étrangers, des laboratoires publics ou privés. 


\section{R.W. Brown}

Physics Department, Case Western Reserve University, Cleveland, OH 44106, U.S.A.

Résumé - Nous décrivons les développements récents de la théorie de perturbation qui résultent de calculs faits sur des réactions avec des bosons de gauge.

Abstract - We describe what we have learned in recent years about perturbation theory as a result of doing calculations for processes with gauge bosons.

\section{I - INTRODUCTION}

During the past half-dozen years many of us have been doing higher-order Born amplitude calculations for quark-gluon processes and for weak-boson production. Among the motives are the interest in multi-jet phenomena in the former case and tests for trilinear couplings in both cases.

Bonuses have been earned from these labors. Simple results corresponding to unforeseen symmetries have been found, despite complicated steps, that tell us that we may very well profit by a reformulation of the vector-boson helicity bases and the associated amplitude decomposition with which we start.

The simple results are seen in both analytical work and numerical computations. We describe the analytical discoveries in terms of three miracles, in the discussion to follow, and we can relate the numerical and analytical results in a unified approach. This approach and certain new conclusions are due to a collaboration involving J. Donohue, M. Karlsson, G.T. Kleppe and myself.

\section{II - HELICITY RULES}

\section{A) Helicity conservation}

Every child in Marseilles knows that a massless spin-1/2 particle preserves its helicity throughout any series of vector/axial-vector (V/A) couplings. With mass, helicity flip is possible but the high-energy limit is smooth since there is no reduction in the number of spin states.

Massless spin- 1 particles also partake of helicity consexvation in a process such as a compton reaction. This is manifest, for both QED and QCD, in the forms of the Compton amplitude to be discussed in due course. That gauge bosons with mass follow suit at high energy is expected at first glance because renormalizable theories are advertised to make a smooth transition from the 3 spin states to the 2 massless spin states.

One may wonder, however, about helicity conservation for vector bosons with mass since unitarity constraints do not generally require that the longitudinal-helicity cross sections vanish relative to the positive-helicity (right-handed $=\mathrm{RH}$ ) and negative-helicity (left-handed $=\mathrm{LH}$ ) rates. We have in mind both non-renormalizable theories and composite vector particles. In fact, angular momentum conservation appears to force one vector particle to be in a longitudinal state in the forward Compton Born amplitude for V/A couplings. Also, the high-energy limits may well depend on which helicity basis ( $s, t, u$ channel, etc.) is utilized for nonzero mass. The näive expectations are realized, however, and we will see presently how elegantly the non-Abelian theories follow the Abelian theories that in turn follow the massless conservation rules. 


\section{B) Helicity correlation}

What is the relation of the vector-boson helicity to the emitting/absorbing Dirac fermion? Years ago, McVoy and Dyson / $1 /$ calculated that electrons "transferred" their helicity to hard photons in bremsstrahlung. We see an analog $/ 2 /$ in the ee $\rightarrow \gamma$ differential cross sections for a given electron handedness (Fig. 1). A LH $\gamma$ is found in the $\mathrm{LH}-\mathrm{e}^{-}$hemisphere and a RH $\gamma$ is found in the $\mathrm{RH}-\mathrm{e}^{+}$hemisphere. We define the hemisphere dominance of helicity-transfer or handedness-transfer as spinoff, an expected occurrence when a propagator pole is present at the phase space boundary. [The pole also explains the helicity conundrum referenced earlier: The angular-momentum zeros, $0(\theta)$, from helicity non-conservation $(1 \neq 2)$ in the forward and backward directions are dominated by $t$-channel and $u$-channel poles, $0\left(\theta^{-2}\right)$, respectively.] Spinoff is quantitatively useful, even when the pole infinity is avoided by, say, an angular cutoff $\theta_{c}$. The error in characterizing the polarization is roughly $O\left(\theta_{c}\right)$.

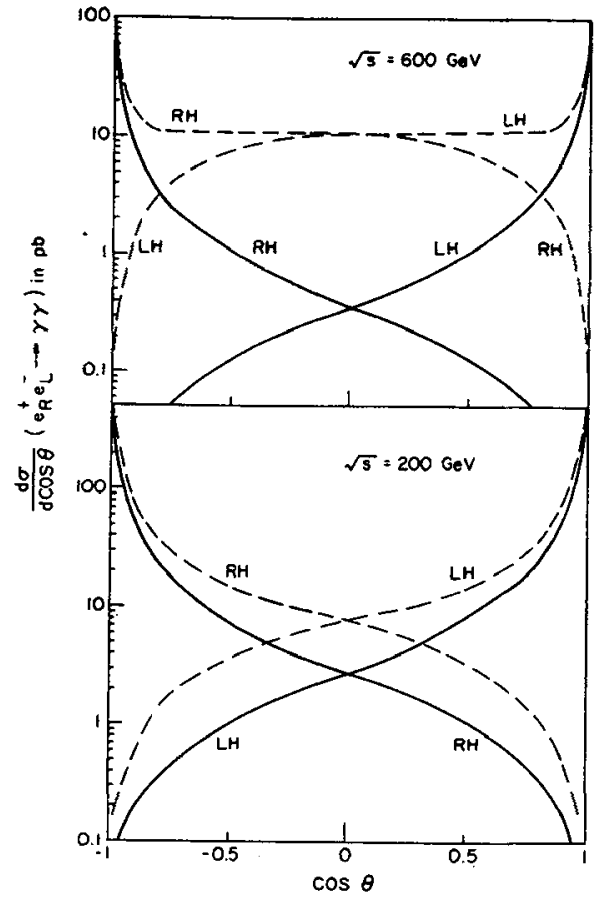

Fig. 1 - Photon polarization for $\mathrm{e}_{\mathrm{RH}}^{+} \mathrm{e}_{\overline{\mathrm{L}} \mathrm{H}} \rightarrow \mathrm{r}$ from $/ 2 /$. The dashed curve corresponds to a nonrenormalizable coupling.

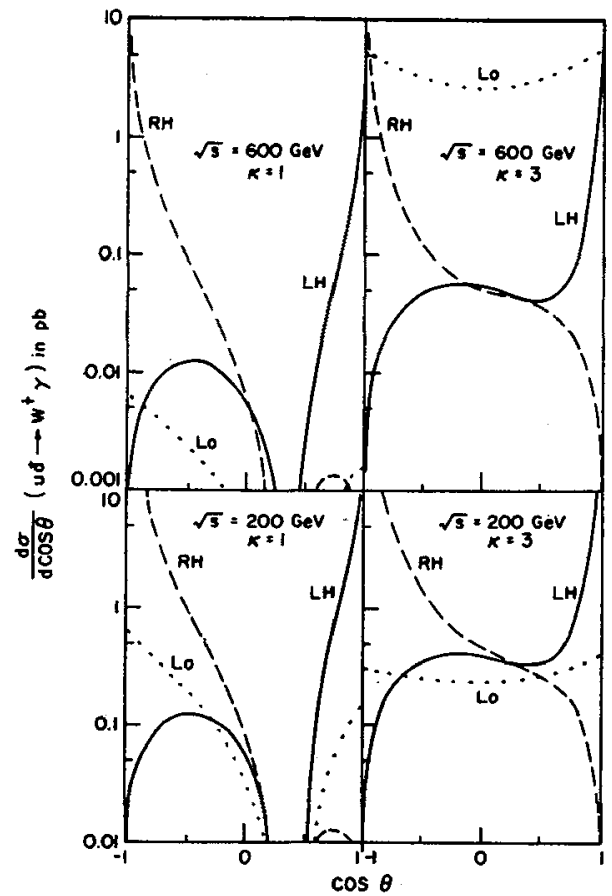

Fig. $2-\mathrm{W}^{+}$polarization for $u d \rightarrow \mathrm{W}^{+} \gamma$ from $/ 2 / . \theta=$ c.m. angle between $W^{+}$ and $u$.

Spinoff applies as well to the Born production of pairs of vector bosons with mass in the annihilation of Dirac fermions,

$$
\vec{f}^{\prime}+V^{\prime} \text {. }
$$

or in its crossed version (Compton). While peaking is certainly seen in the earlier calculations of Gaemers and Gounaris /3/ and of Hellmund and Ranft /4/, these 
authors employed a rectangular polarization basis, so we turn to Bilchak et al. $/ 2 /$ who directly addressed $s$-channel helicity transfer in ee $\rightarrow \gamma \gamma, Z \gamma, W W, Z Z$ and $\mathrm{q} \overline{\mathrm{q}}^{\prime} \rightarrow \mathrm{W}^{\mathrm{N}}, \mathrm{Z \gamma}, \mathrm{Wg}, \mathrm{Zg}, \mathrm{WW}, \mathrm{WZ}, \mathrm{ZZ}$.

For $u d \rightarrow w^{+} \gamma\left(/ 2 /\right.$ and Fig. 2) there is $80 \%(20 \%) W^{+}$spinoff from $\bar{a}(u)$ in agreement with the squared quark charge ratio. This is true even near threshold. By contrast the longitudinal contribution dominates, as the energy is increased, for nonrenormalizable couplings (e.g., the $k=3$ curves).

Figures 3 and 4 show results for pair production where both vectors have mass and where spinoff applies only away from threshold, with two-pole enhancement for ee $\rightarrow \mathrm{ZZ}$ and one-pole enhancement for ee $\rightarrow \mathrm{WW}$. The scale in the figures is sometimes too coarse to show the structure at forward and backward angles that results from the kinds of angular momentum constraints discussed previously.

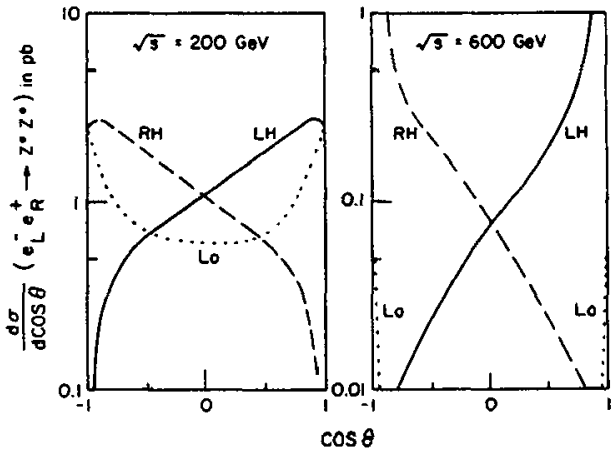

Fig. $3-z^{0}$ polarization for $\mathrm{e}_{\mathrm{LH}}^{-} \mathrm{e}_{\mathrm{RH}}^{+} \rightarrow \mathrm{z}^{\circ} \mathrm{Z}^{\circ}$ from $/ 2 /$.

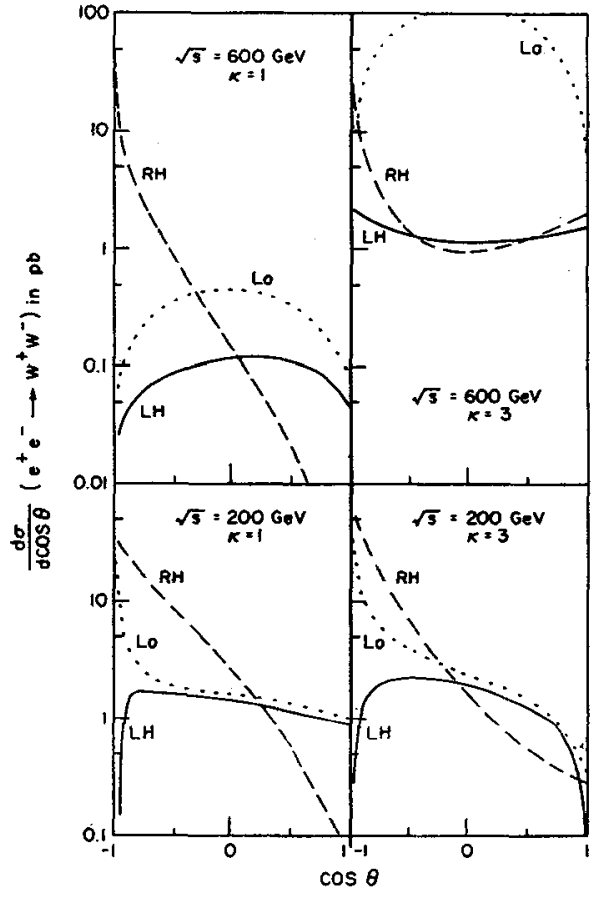

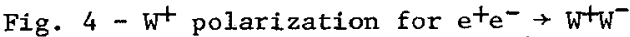
from $/ 2 / . \theta=c . m$. angle between $W^{+}$and $e^{-}$.

The relevance of spinoff is not obvious since the longitudinal helicity state permits forward/backward angular momentum conservation and since a trilinear-boson coupling contributes to $\mathrm{W} \gamma$ and $W W$ production. Indeed spinoff is spoiled for nongauge $W$ couplings, as seen in the figures. We expect that the longitudinal helicity state complicates matters for nonrenormalizable interactions, even when the necessary unitarity corrections are made, or for the production of composite vector bosons. In general, renormalizable gauge theories are required for spinoff to apply to Born amplitudes.

It is satisfying that spin results for other vector-boson reactions can be described similarly. The weak bosons spin off the neutrino in the classic reaction for $W$ 
searches, $\nu z \rightarrow \ell W z^{\prime}$, and off the initial charged lepton in $\ell z \rightarrow \ell z z^{\prime}$ but not in $\ell z \rightarrow V_{W} z^{\prime} / 5 /$. A cross-channel lepton exchange is present in the first two compton subamplitudes but not in the third. The integration over the virtual photon, from the electromagnetic recoil off the nucleus $z$, does bring out differences in detailed comparisons, such as a decrease in spinoff as the $l z$ laboratory energy is increased.

No pole is present in the important charge asymmetry $/ 6 /$ in $\bar{p} \bar{p} \rightarrow W X$, which is the tendency of the decay lepton (antilepton) to follow the $p(\bar{p})$. (The effects of polarized proton beams on the asymmetry are detailed in a contribution to this conference by P. Chiapetta and J. Soffer.) But we still may say that the $\mathrm{w}^{+}$spins off $u$, when $i t$ is found in the proton hemisphere, backward biasing the $e^{+}$, and so forth.

\section{III - HELICITY BASES}

\section{A) CALKUL Miracle}

The simple conservation and spinoff rules suggest that we search for vector helicity bases that most directly exhibit the rules analytically. The CALKUL basis /7/ (Cambridge, Leiden, Katholieke Univ. Leuven) is just what we need both to simplify massless calculations and to connect with spinoff. We define a basis for an outgoing $\mathrm{RH}(+)$ or $\mathrm{LH}(-)$ photon with respect to two spinor momenta $\mathrm{p}, \mathrm{p}^{\prime}$ :

$$
\begin{aligned}
& \varepsilon^{ \pm}(q)=\sqrt{\frac{\pi}{2}}\left(\varepsilon^{\prime \prime}+i \varepsilon^{\perp}\right), \\
& \varepsilon_{\dddot{\prime}}^{\prime \prime}=N \sqrt{\frac{\pi}{2}}\left(p \cdot q p_{\mu}^{\prime}-p^{\prime} \cdot q p_{\mu}\right), \\
& \varepsilon_{\mu}^{\perp}=N \sqrt{\frac{\sqrt{2}}{2}} \varepsilon_{\mu}^{\alpha \beta \gamma} p_{\alpha} p_{\beta}^{\prime} q_{\gamma}, \\
& N=\left(p \cdot p^{\prime} p \cdot q p^{\prime} \cdot q\right)^{-\frac{1}{2}} .
\end{aligned}
$$

With identities such as

$$
\not^{ \pm}=\frac{N}{4}\left[p^{\prime} \phi\left(1 \mp \gamma_{5}\right)-\not \phi^{\prime} \not\left(1 \pm \gamma_{5}\right) \pm 2 p \cdot p^{\prime} \not \gamma_{5}\right] \text {, }
$$

we benefit from the disappearance of many terms due to chirality mismatch, masslessness, and current conservation, for photon attachments to a vertex $\Lambda_{\mu}\left(p^{\prime}, p\right)=$ $\gamma_{\mu}, \gamma_{\mu} \gamma_{5}$. The phase differences from vertex to vertex are readily calculated and the terms that remain simplify significantly.

Besides the miraculous simplification of high-order Born amplitudes, such as for ee $\rightarrow r r r$, we also achieve a quick grasp of spinoff. The chiral massless fermion with four-momentum $p$ has the polarization limit $s^{\mu} \propto \pm p^{\mu}$. Thus the photon attached to either side, yielding a Compton subprocess, has its polarization determined by $\mathrm{p}, \mathrm{p}^{\prime}$ and the \pm sign. The secret of CALKUL - the circularity and the use of the source parameters is in turn the secret of spinoff. A precise pole - helicity connection for the Compton amplitude is made in Sec. IV.

\section{B) CALKUL for mass}

If only one vector boson has mass in (1) or in its crossed (Compton) version, then the CALKUL basis is readily generalized to represent that boson. Putting the masslessness of the other boson to good use and adding a longitudinal component, we can make an easy analytical demonstration of longitudinal suppression, and of spinoff with the adapted s-channel basis $/ 2 /$. An important step is the implementation of radiation symmetry, a point to which we will return.

Passarino /8/ has employed the Dirac spinor formalism of Caffo and Remiddi /9/ to obtain a general representation for vector polarization bases. The idea is to use 
Bargmann-Wigner formulas to write a wave function for a vector boson with mass $m$,

$$
\varepsilon^{\mu}=\frac{1}{4 m} \sum_{\text {symm }} \bar{v} \gamma^{\mu} u \text {, }
$$

in terms of spinors defined according to $/ 9 /$. The use of the fermion coordinates present in a scattering problem is clearly suggested by (7) the limit of which is fundamentally related to the CALKUL formula. Simpler calculations ensue, even when no particle is massless.

\section{IV - HELICITY AMPLITUDES}

\section{A) Donohue Miracle}

The ultimate step that should be taken to make manifest the helicity rules is to decompose the Feynman Born amplitudes into the appropriate helicity basis. If there were helicity states that did not contribute at all, to take a simple example, their omission would be clear at the outset. The question is, are there other bases for vector bosons with mass, besides the s-channel ones under discussion, where the amplitudes are even simpler?

Consider as a building block the Abelian (no trilinear coupling) Compton reaction,

$$
V f \rightarrow V^{\prime} f^{\prime}
$$

with massless Dirac fermions and both vector masses nonzero. A crossing argument connects our conclusions to (1). Donohue $/ 10 /$ found the surprising result that the Born amplitude for (8) has a $2 \times 2$, rather than $3 \times 3$, form,

$$
M=M_{1}\left|V^{\prime}, 1^{s}\right\rangle\left\langle v, 1^{s}\left|+M_{-1}\right| V^{\prime},-1^{u}\right\rangle\left\langle v,-1^{u}\right|
$$

for $R H f^{\prime} f^{\prime}$. LH $f, f^{\prime}$ require the replacement $1 \leftrightarrow-1$ for the vector helicities. The superscripts $s, u$ refer to the helicity channel.

By the removal of prejudice against non-orthogonal bases, we find ourselves with only two terms, one connecting s-channel helicities and another u-channel helicities in diagonal form and with one spin dimension missing. The non-orthogonality implies that helicity is not conserved but if, for example, $V$ is in $\left|+l^{\prime}\right\rangle$ then $V^{\prime}$ must be in $\left|+1^{s}\right\rangle$ and so on. Pair production (1) likewise has a diagonal form obtained by crossing $(s \rightarrow t)$. The $V, V^{\prime}$ bosons may be virtual with helicities defined as in deep inelastic electron scattering for the spacelike case.

The secret of Donohue's representation is related to the success of CALKUL - we can show that the Donohue basis reduces to the CALKUL basis obtained by dropping the $₫ \gamma_{5}$ term in (6). The differences between the helicity frames disappear in the massless limit. We have a gauge-invariant decomposition into two radiation poles whose residues are pure helicity projection operators with the channel and sense determined by the parent spinor. The spinoff observed for ee $\rightarrow \mathrm{ZZ}$ is thereby derived, noting that the forward and backward scattering limits of the orthogonal $s$-channel decomposition of Fig. 3 coincide with the respective $t, u$ singularities in the crossed form of (9). (We obtain s-channel longitudinal contributions from \pm 1 helicity states in the $t, u$ channels.) As another example, analysis of the singularities in (9) shows that $V^{\prime}$ spinning off $f$ is to be expected for (8).

\section{B) Mikaelian Miracle}

We consider next a non-Abelian Compton reaction (8) where a trilinear coupling exists for the two vector bosons at least one of which is massless. An example is the photoproduction of a weak boson off quarks or electrons,

$$
Y f^{\prime} \rightarrow \mathrm{Wf}^{\prime}
$$


Sometime ago Mikaelian /11/ found that the unpolarized differential cross section for (10) could be written in factorized form with perfectly squared coefficient, but only for the gauge theory value of the $W$ magnetic moment. This was eventually beatified by the amplitude analysis (Born Again) of Goebel, Halzen and Leveille/12/.

It is helpful to digress to discuss the symetry underlying this factorization. The crossed reaction to $(10)$,

$$
\mathrm{f} \overline{\mathrm{f}} \cdot \mathrm{WY},
$$

has been suggested as a probe of the $W$ couplings and has related factorization /13/. Mikaelian, Samuel and Sahdev /14/ showed that the factor could produce zeros at certain c.m. angles in the differential cross sections for (11). [The zeros can also be seen in the curves of $/ 13 /$ and in Fig. 2.] It remained, however, for Goebel et al. to recognize and develop the implied universal factorization of each 4-body helicity amplitude.

Brodsky, Kowalsky and Brownsky have discovered that factorization is a special case of a general radiation symmetry, which can be connected back to classical interference effects and which leads to spin-independent zeros (often unphysical) in all gauge-theory Born amplitudes (spins $\leq 1$ ) for gauge boson emission/absorption /15/. (It is amusing that we had to wait for $W$ calculations to stimulate the discovery of a symmetry that is present in a Jackson problem!) There is also a complementary theorem /15,16/ for spin-dependent symmetry and associated spin-dependent zeros. Generalizations to an all-orders external plane-wave decoupling theorem /17/ and to gaugino emission in supersymmetry /18/ now exist.

Returning to the helicity analysis, we use what we have learned from /12/ to write the non-Abelian 4-body amplitude in the simple form

$$
\mathrm{M}_{\mathrm{NA}}=\mathrm{Z}_{\mathrm{A}}
$$

$Z$ is the spin-independent coefficient in which the radiation zero resides and $M_{A}$ is an Abelian Compton amplitude. Various forms are possible by virtue of the radiation symmetry /15/. Namely, the general Born $(n+1)$-body tree radiation amplitude (particles with charges $Q_{i}$, momenta $p_{i}$; photon momentum $q$ ) can be written

$$
\begin{aligned}
& M=\sum_{i=1}^{n} \frac{Q_{i} J_{i}}{p_{i} \cdot \mathrm{q}}, \\
& \sum Q_{i}=\sum \mathrm{p}_{i} \cdot \mathrm{q}=\sum \mathrm{J}_{i}=0
\end{aligned}
$$

The expression (13) is invariant under

$$
Q_{i} \rightarrow Q_{i}+\alpha p_{i} \cdot q
$$

and/or

$$
\mathrm{J}_{i} \rightarrow \mathrm{J}_{i}+\beta \mathrm{p}_{i} \cdot \mathrm{q}
$$

Whatever, (12) is what we want. With the trilinear coupling transformed away (really, no longer explicit), we may immediately apply the Donohue form (9) to $\mathrm{M}_{\mathrm{A}}$ and hence, $\mathrm{M}_{\mathrm{NA}}$. The Donohue -Gottlieb result $/ 10 /$ suffices, actually, since one vector is massless. The final formula achieved makes manifest the radiation symmetry and the spin symmetry (helicity rules), calculations with which are particularly simple. Contrast should be made with the procedure of squaring and spin summing where terms proliferate and polarization information is lost. We may combine the technique of Bjorken and Chen /19/ for projecting out arbitrary polarizations with our approach. 


\section{More particles}

A Born amplitude for the radiation of (at least) one massless gauge boson by Dirac particles may be regarded as the sum of graphs generated by attaching the gauge boson in all possible ways to a set of source graphs. The set of graphs may be rearranged as a gauge-invariant vertex expansion where the attachments are clustered at each vertex /15/. An internal line attachment leads to two radiation factors, one for each associated vertex. Then for each 3 -vertex we have generated a Compton set of subgraphs to which we can apply a helicity form, utilizing the factorization (12) in the event that there is a non-Abelian trilinear attachment involved. of course, the helicity channels are now defined relative to the individual legs, external and internal, but this is expected from spinoff.

In general, (12) is not realized for $n$-body Born radiation amplitudes, $n \geq 5$. We wish to emphasize that the symmetry under (15) and (16) reduces (13) to (12) only for $n=3$ and that radiation zeros are the general result, not factorization.

We make note, however, of the 5-body factorization discovered by CALKUL /7/ in the Born amplitude for $f \bar{f} \rightarrow f^{\prime} \bar{f}^{\prime} \gamma$, a result that rests on the fermions being massless. Fermion helicity conservation is presumably crucial, since scalar vertices spoil the factorization. This additional symetry may be related to a combination of radiation symmetry, which only reduces the amplitude to a two-dimensional zero, and the helicity form for the Compton subgraphs (one vector is the exchanged virtual photon).

\section{More masses}

In the circumstance where both vector bosons have mass, real or virtual, the nonAbelian Compton factorization (12) is no longer possible. MA reverts to $3 \times 3$ form, though it is still diagonal in terms of the three independent polarization vectors introduced in /10/. The third diagonal matrix element vanishes relative to the two terms that are analogous to those in (9), as energy is increased. At high energies, non-Abelian Compton Born amplitudes go over to Abelian forms that in turn are expressed more clearly with a non-orthogonal basis.

\section{$\mathrm{V}$ - SPIN DEPENDENT ZEROS}

Besides the spin-independent zeros that arise in charge-dependent phase-space regions, corresponding to equal $Q_{i} / p_{i} \cdot q$ [see (15)], we have spin-dependent zeros that arise in charge-independent ways, corresponding to equal $\mathrm{J}_{i} / \mathrm{p}_{i} \cdot \mathrm{q}$ [see (16)] and residing, for example, in $M_{A}$ rather than in $Z$ for (12). This is the complementary radiation theorem for gauge theories that we have already introduced.

An interesting instance of a spin- dependent zero is the complete vanishing of the Born amplitude for (11) whenever the photon is polarized transverse to the scattering plane and the $W$ is longitudinal $/ 20,4,16 /$. Nongauge couplings or photons with mass give a nonzero answer. This and other examples suggest ways to measure selected helicity contributions or to test gauge theories.

We might ask how these zeros are related to the Donohue form, which itself predicts zeros in helicity amplitudes. Regarding the form as a linear functional $D \cdot \varepsilon$ for photon polarization vector $\varepsilon$ and a given $W$ polarization in (11), there is a direction $\varepsilon_{\mathrm{n}}$ that yields a null functional, $\mathrm{D} \cdot \varepsilon_{\mathrm{n}}=0$. When $\varepsilon_{\mathrm{n}}$ is physical we obtain a spin-dependent radiation zero. This is not, however, the zero on the diagonal of the matrix obtained from the non-orthogonal bases.

VI - OUTLOOK

We have not yet progressed to the point where a recipe is available for the ready simplification of arbitrary tree gauge-theory amplitudes with arbitrary masses. 
Closed-loops, about which we will comment shortly, present further problems. Nevertheless the view of vector-boson amplitudes as a series of attachments, generating an expansion of pole terms with specific helicities, should continue to be useful, perhaps in concert with a formalism such as that of Passarino /8/. We have made progress on the high-energy limit of an arbitrary Born amplitude, where the masses are neglected. In that limit we can make the radiation vertex expansion, eliminate certain trilinear couplings by radiation symetry, and then decompose into CALKUL/Donohue poles. The CALKUL decomposition is especially simple, using identities like (6), for the Abelian Compton subgraphs. Any sharp value of helicity found in a particular phase-space region is invariably correlated with a pole enhancement (spinoff).

The desiderata also include the need to consider the helicity analysis of Compton scattering off other vector particles. Kim and Tsai have shown that the helicity of $\mathrm{a} \mathrm{W}$ is conserved in the scattering from an electronagnetic field but on ly at small angles /21/. More information comes from a contribution to this conference by V.A. Koval'chuk and I.V. Stoletnil. We interpret their density-matrix computation for $\gamma e \rightarrow W$ as indicating that the $W$ helicity matches that of the $\gamma$ in the dominant forward peak. This is spinoff associated with a t-channel pole. But further work on the general $\vec{V}_{1} V_{2} \rightarrow V_{3} V_{4}$ Born helicity treatment analogous to Donohue's research should be done.

For completeness, we notice that spin-zero particles $\sigma$ are easily included and fit nicely in to the general picture. For example, the poles in the Vo $\rightarrow$ Vo amplitude and its crossed channels do not lead to sharp vector helicities - the calculations using (2) - (5) are simple here - unless it is $V$ spinning off $V$. We cannot spin off a scalar particle.

Open and interesting questions revolve around connections to other contributions to this symposium. It remains to be seen if vector helicity bases effective in Born amplitudes are useful in calculations such as that by $M$. Anselmino and P. Kroll on photoproduction of vector mesons or in the program to test dynamical models by G.R. Goldstein and M.J. Moravcsik. In addition, it may be possible to combine the Weyl representation involving two-component spinors, where Fierz identities bring substantial streamlining to the given problem (see, e.g., /22/), with what we have to say.

It may be possible to simplify closed-loop calculations because of the symmetries we have discussed. For instance, the radiation symetry under (15) and (16) can be extended to closed loops provided that the internal charges and currents are shifted as well. Perhaps an off-shell version of this would lead to cancellations in higher-order QED radiative corrections.

Let us not lose sight, finally, of a central application of our work. The identification of the possible poles and their control of helicity yields a quantitatively useful estimate of vector-boson decay distributions. The details of the demise of weak bosons are a major concern at the present time.

\section{ACKNOWLEDGMENT}

I am most grateful to Professor J. Soffer, to the symposium committee, to my collaborators (C. Bilchak, J. Donohue, M. Karlsson, G. Kleppe and J. Stroughair), and to the National Science Foundation (USA) for making this progress report possible. I thank $\mathrm{K}$. Kowalski for helpful research discussions and criticism. 


\section{REFERENCES}

/I/ McVOY K.W. and DYSON F.J., Phys. Rev. 106 (1957) 1360.

/2/ BILCHAK C.L., BROWN R.W. and STROUGHAIR J.D., Phys. Rev. D29 (1984) 375; BROWN R.W., Proton-Antiproton Collider Physics-1981, edited by V. Barger, D. Cline, and F. Halzen (AIP, New York, 1983), p. 251.

/3/ GAEMERS K.J.F. and GOUNARIS G.J., 2 Phys. Cl (1979) 259.

14 HELLMUND M. and RANFT G., Z Phys. C12 (1982) 333.

15/ BELL J.S. and VELTMAN M., Phys. Le tt. 5 (1963) 151; ÜBERALl H., Phys. Rev. 133 (1964) B444; REIFF J., Nucl. Phys. B23 (1970) 387; BROWN R.W., HOBBS R.H. and SMITH J., Phys. Rev. D4 (1971) 794; BROWN R.W., GORDON L.B. and MIKAELIAN K.O., Phys. Rev. Lett. 33 (1974) 1119; GORDON L.B. and BROWN R.W., Phys. Rev. DI2 (1975) 2851.

16/ CLINE D.B., RUBBIA C. and VAN DER MEER S., The Search for Intermediate Vector Bosons, Scientific American (1982) March.

/7/ BERENDS F.A., KLEISS R., DE CAUSMAECKER P., GASTMANS R., TROOST W. and WIT T.T., Nuc1. Phys. B206 (1982) 61, references therein and subsequent preprints.

$18 /$ PaSsarino G., Nucl. Phys. B237 (1984) 249.

/9/ CAFFO M. and REMIDDI E., Helv. Phys. Acta 55 (1982) 339.

$/ 10 /$ DONOHUE J.T., Phys. Rev. D29 (1984) 393; DONOHUE J.T. and GOTTLIEB S., Phys. Rev. D23 (1981) 2581.

/1I/ MIKAE LIAN K.O., Phys. Rev. D17 (1978) 750.

112/ GOEBEL C.J., HALZEN F. and LEVEILLE J.P., Phys. Rev. D23 (1981) 2682; ZHU D., Phys. Rev. D22 (1980) 2265.

/13/ BROWN R.W., SAHDEV D. and MIKAELIAN K.o., Phys. Rev. D20 (1979) 1164.

/14/ MIKAELIAN K.0., SAMUEL M.A. and SAHDEV. D., Phys. Rev. Lett. 43 (1979) 746.

/15/ BRODSKY S.J. and BROWN R.W., Phys. Rev. Lett. 49 (1982) 966; BROWN R.W., KOWALSKI K.L. and BRODSKY S.J., Phys. Rev, D28 (1983) 624; BROWN R.W., Electroweak Effects at High Energies, proceedings of the Europhysics Study Conference, Erice, Italy, 1983, edited by H. NEWMAN (Plenum, New York, 1984); See also SAMUEL M.A., Phys. Rev. D27 (1983) 2724.

/16/ BROWN R.W. and KOWALSKI K.L., Phys. Rev. D29 (1984) 2100.

/17/ BROWN R.W. and KOWALSKI K.I., Phys. Rev. Lett. 51 (1983) 2355 and Phys. Rev. D30 (1984) in press.

/18/ BROWN R.W. and KOWALSKI K.L., Phys. Lett. 144B (1984) 235; BARGER V., ROBINETT R., KEUNG W.Y. and PHILlIPS R.J.N., Phys. Lett. 131B (1983) 372; ROBINETT R., Phys. Rev. D30 (1984) 688.

/19/ BJORKEN J.D., and CHEN M.C., Phys. Rev. 154 (1967) 1335.

$120 /$ CORTÉS J., HAGIWARA K. and HERZOG F., Phys. Rev. D28 (1983) 2311.

/21/ KIM K.J. and TSAI Y.-S., Phys. Rev. D7 (1973) 3710.

/22/ FARRAR G.R. and NERI F., Phys. Lett. 130B (1983) 109. 